\title{
Review \\ Pro/con debate: Should synthetic colloids be used in patients with septic shock?
}

\author{
James Downar ${ }^{1}$ and Stephen E Lapinsky ${ }^{2}$
}

\begin{abstract}
${ }^{1}$ Divisions of Critical Care and Palliative Medicine, Department of Medicine, University of Toronto, 190 Elizabeth Street, Toronto M5G 1X5, Canada 2Intensive Care Unit, Mount Sinai Hospital, 600 University Ave, Toronto M5G 1X5 and Interdepartmental Division of Critical Care, University of Toronto, 30 Bond Street, Toronto, M5B 1W8, Canada
\end{abstract}

Corresponding author: Stephen E Lapinsky, stephen.lapinsky@utoronto.ca

Published: 29 January 2009

This article is online at http://ccforum.com/content/13/1/203

(c) 2009 BioMed Central Ltd
Critical Care 2009, 13:203 (doi:10.1186/cc7147)

tain more molecules of HES for a given concentration and thus have a higher oncotic pressure, but they have a shorter half-life in vivo because they are more quickly broken down by serum amylase to $50-\mathrm{kDa}$ molecules that can be excreted in the urine. Solutions are typically divided into three weight categories: high MW (approximately $450 \mathrm{kDa}$ ) (for example, Hespan ${ }^{\circledR}$ ), medium MW (200 to $260 \mathrm{kDa}$ ) (for example, HemoHES ${ }^{\circledR}$ and Pentaspan ${ }^{\circledR}$ ), and low MW (70 to $130 \mathrm{kDa}$ ) (for example, Voluven ${ }^{\circledR}$ ).

To slow metabolism by amylase, HES molecules have hydroxyethyl radical groups substituted onto individual glucose units. The degree of hydroxyethyl substitution is expressed by the molar substitution ratio, which is simply a ratio of the number of substituted glucose molecules to the total number of glucose molecules. Highly substituted HES solutions have a ratio of 0.6 to 0.7 and are metabolized slowly. Less substituted HES solutions have a ratio of 0.4 to 0.5 and are metabolized quickly. Finally, the point of attachment of the hydroxyethyl group is also important. Hydroxyethyl groups attached at the C2 position on the glucose ring slow metabolism more than those attached at the $\mathrm{C} 6$ or $\mathrm{C} 3$ position. Thus, a high $\mathrm{C} 2 / \mathrm{C} 6$ ratio $(>8)$ slows metabolism more than a low $\mathrm{C} 2 / \mathrm{C} 6$ ratio $(<8)$.

\section{Gelatins}

Gelatins are polypeptides derived from bovine collagen. Modern gelatin preparations are chemically modified in various ways to reduce viscosity while maintaining their oncotic effect. Gelatins are smaller molecules than HESs (approximately $35 \mathrm{kDa}$ ) and therefore are more rapidly broken down and eliminated. However, there is no published dose limitation for gelatins as there are for HES and dextrans [2].

$\mathrm{ARF}=$ acute renal failure; HES = hydroxyethyl starch; MW = molecular weight; VISEP = Efficacy of Volume Substitution and Insulin Therapy in Severe Sepsis. 


\section{Dextrans}

Dextrans are a polysaccharide mixture derived from the bacterium Leuconostoc mesenteroides, typically used in solutions of either 40- or 70-kDa molecules. Dextrans have a high water-binding capacity and are very effective for reducing blood viscosity [3].

\section{Pro: There is no justification for using synthetic colloids in patients with septic shock}

Investigators have long known about the potential risks of synthetic colloids, including renal failure, coagulopathy, anaphylactoid reactions, reticuloendothelial dysfunction, hepatic dysfunction, and severe pruritus [4]. As described in the section above, the synthetic colloids represent a group of products with different properties, and studies of one product may not be representative of the group as a whole. However, numerous investigators have concluded that synthetic colloids are safe (or superior to albumin or crystalloids) on the basis of small studies using surrogate, biochemical, or hemodynamic endpoints and often very brief follow-up [5-15].

On the other hand, two large prospective randomized trials with lengthy follow-up and hard endpoints have now shown that HES is associated with a significantly increased risk of acute renal failure (ARF), coagulopathy, and possibly mortality in patients with septic shock. Schortgen and colleagues [16] randomly assigned 129 patients to receive either HES $200 / 0.62$ or $3 \%$ gelatin for intravascular volume expansion and followed them for 34 days. They found that the HES group had a significantly higher incidence of ARF (defined as a twofold increase in creatinine, $42 \%$ versus $23 \%$; $P=0.028$ ), and in a multivariate analysis, the use of HES was associated with an odds ratio of 2.57 for ARF. The investigators were careful to respect the dose limitations recommended by the manufacturer, giving an average of $14 \mathrm{~mL} / \mathrm{kg}$ (range of 10 to 26) on day 1 and less than $20 \mathrm{~mL} / \mathrm{kg}$ for the next 3 days. HES was not administered after the fourth day.

In the more recent VISEP (Efficacy of Volume Substitution and Insulin Therapy in Severe Sepsis) study, Brunkhorst and colleagues [17] randomly assigned 537 patients with severe sepsis or septic shock to receive either HES 200/0.5 or Ringer lactate as part of a 96-hour protocol to maintain a central venous pressure of greater than $8 \mathrm{~mm} \mathrm{Hg}$, a central venous $\mathrm{O}_{2}$ saturation of greater than $70 \%$, and a mean arterial pressure of greater than $70 \mathrm{~mm} \mathrm{Hg}$. Again, patients in the HES group had a higher incidence of renal failure requiring dialysis (31.0\% versus $18.8 \% ; P=0.001)$. The protocol specified a daily limit of $20 \mathrm{~mL} / \mathrm{kg}$ of HES [17] but this was exceeded in approximately $35 \%$ of patients in the HES group, mostly on day 1 . When the investigators looked only at the patients who had received less than $20 \mathrm{~mL} / \mathrm{kg}$ of study fluid daily, there was still a significantly higher incidence of renal failure requiring dialysis. The investigators also identified a clear dose effect of HES. Higher cumulative doses of HES were correlated with both a higher 90-day mortality rate and a higher incidence of renal failure requiring dialysis. This dose effect was not seen in the Ringer lactate group.

While other investigators did not detect a significant increase in the incidence of ARF or mortality, this may have been due to their shorter follow-up (that is, less than 5 days). Data from the latter two studies suggest that differences in the incidence of ARF and mortality may not become apparent until 5 and 30 days, respectively [18].

HES administration is also associated with a deterioration in renal function in patients undergoing cardiovascular surgery [9,19] and kidney donation [20]. The mechanism of renal injury from HES is unclear, but possible explanations include osmotic injury [20], ischemic injury from hyperviscosity [21], or injury related to significant HES uptake by the reticuloendothelial system in the kidney $[22,23]$.

Some have argued that the increased incidence of renal failure can be attributed to either the long half-life or crystalloid solvent of some HES solutions, suggesting that newer lowMW HES [24] or 'balanced' solvents [25] might be safe. However, studies of low-MW HES in cardiac surgery patients show evidence of impaired postoperative glomerular filtration rate [9] and elevated serum markers of renal function [19], and patients who received low-MW HES during abdominal surgery showed greater elevations in $\alpha 1$-microglobulin levels than those who received medium-MW HES [10]. Furthermore, a study comparing a 'balanced' and 'unbalanced' HES solution in patients undergoing abdominal surgery demonstrated no difference, with no renal dysfunction or coagulopathy in either group [6]. Thus, the advantages of low-MW HES or 'balanced' solvents are certainly not proven [6].

There are no large, published, randomized controlled trials of dextrans or gelatins in patients with sepsis, but the data from other clinical settings are not encouraging [26]. In patients undergoing cardiac surgery, gelatin shows an effect similar to HES on markers of renal dysfunction [19] and is associated with higher rates of ARF than crystalloid [27] and HES [28]. Studies of dextrans also show an association with renal failure [29,30]. There is no evidence to suggest that gelatins and dextrans are associated with a lower risk of renal dysfunction than crystalloid.

High- and medium-MW HES preparations cause coagulopathy primarily by reducing levels of factor VIII and von Willebrand factor and by interfering with platelet function [24,31]. In the VISEP study, patients in the HES group developed worse coagulopathy, as demonstrated by a higher Sequential Organ Failure Assessment (SOFA) coagulation score, and a greater need for transfusion of packed red blood cells compared with the crystalloid group (median of 6 units versus 4 units) [17]. Low-MW HES preparations do not appear to show the same effects on factor VIII and von Willebrand factor [24]. Again, fewer studies have examined 
the bleeding risks associated with dextrans and gelatins but both appear to be associated with impaired coagulation and increased bleeding risk [2,32].

Synthetic colloids are effective volume expanders but their use in septic shock cannot be justified in light of the adverse effects. Even 'safe' doses are associated with significant morbidity; newer generation colloids appear to cause adverse reactions similar to the older colloids, and 'balanced' solvent solutions are unproven. Until a large randomized trial with hard endpoints and adequate follow-up demonstrates that one of these solutions is safe and effective, we must conclude that the only safe dose for synthetic colloids is zero.

\section{Con: Synthetic colloids should be used but not abused}

A policy to ban synthetic colloids in the treatment of critically ill patients with septic shock is certainly not supported by the current data. The argument revolves largely around two randomized controlled trials [16,17], both of which used a clinically unlikely regimen: in the HES arm, the starch appeared to be administered as the sole intravenous fluid for many patients (up to a daily maximum level). In the real-life situation, colloids are usually used as intermittent boluses in conjunction with concomitantly administered crystalloid fluids. A study arm in which colloid was the predominant intravenous fluid provided is not generalizable to usual clinical practice. Furthermore, the dose of colloid administered in the VISEP study was far higher than that used clinically, with a median dose of $2.4 \mathrm{~L}$ (and a maximum dose of $13 \mathrm{~L}$ !) on the first day of therapy [33]. In clinical practice, it is uncommon to administer near the maximal dosage. A further confounding issue is the fact that, in the VISEP study, $26.6 \%$ of patients in the crystalloid arm received some form of colloid, either the study HES or another colloid product [17].

Studies of HES used as an adjunct to crystalloid fluid therapy, to facilitate reaching hemodynamic targets, have shown benefit. A study of septic and trauma patients, in which HES was given with crystalloid (administered in a volume ratio of approximately $1: 3.5)$, showed clear benefits in terms of hemodynamic improvement and oxygen delivery [5]. Long-term renal function was not assessed in this study. The absence of an association of HES with renal failure in usual clinical practice was demonstrated in a large European observational study of over 3,000 patients [34]. One third of these patients $(1,075$ patients) received HES with a median dose of $555 \mathrm{~mL}$ per day (median total dose of $1,000 \mathrm{~mL}$ in 2 days). Although the incidence of renal failure was higher in this group, there was no association between HES and dialysis in a multivariable model. Patients who received HES were also older, were more likely to be surgical patients, and had a higher severity of illness.

Others have reported a lack of association between the use of HES and renal failure in clinical use, in which HES is combined with crystalloid therapy [35]. In a study of 62 patients undergoing abdominal aortic surgery, a reduced incidence of renal injury was noted in patients randomly assigned to received one of two HES products (HES 200/0.62 or HES 130/0.4) compared with gelatin [36]. All patients received concomitant infusions of normal saline, amounting to approximately twice the volume of colloid. Only one patient in each HES group required renal replacement therapy $(4.8 \%)$ compared with three in the gelatin group (15\%). A recent study evaluated renal effects in 33 patients with pre-existing mild renal dysfunction who were randomly assigned to receive $6 \%$ HES (130/0.4) or gelatin (in addition to at least $1.5 \mathrm{~L} /$ day crystalloid) for perioperative volume replacement for abdominal aortic surgery [37]. Five patients (15\%) in the HES arm developed severe renal impairment that was not different from the gelatin-treated arm, although this does not exclude the possibility that both the HES and gelatin caused some renal dysfunction.

Furthermore, a comprehensive ban on all synthetic colloids would overlook the important differences between older and newer synthetic colloids. Neither of the above-mentioned randomized trials studied one of the new low-MW HES solutions. No study has demonstrated a link between lowMW HES solutions and an increased need for dialysis, and while high-MW HES solutions are clearly associated with coagulopathy, the low-MW HES solutions are not [24,38,39]. A study of renal function in cadaveric renal transplant recipients compared donors resuscitated with a 200/0.6 hetastarch and those resuscitated with a new-generation $130 / 0.4$ product in a retrospective matched paired study [40]. The group that received the 130/0.4 hetastarch had a significantly reduced incidence of delayed graft failure $(22 \%$ versus 33\%). Furthermore, animal studies suggest that HES solutions containing a more physiologically 'balanced' solvent (for example, Ringer lactate) might cause less renal impairment and coagulopathy than one containing normal or hypertonic saline [25]. One study in patients undergoing abdominal surgery found that a 'balanced' HES solution produced a smaller acid-base disturbance than an 'unbalanced' solution [6].

The results of Schortgen and colleagues [16] and Brunkhorst and colleagues [17] should warn clinicians not to exceed the recommended daily dose of synthetic colloid and to use a balanced approach to fluid resuscitation that includes crystalloid solutions. They should not serve as the basis of a comprehensive ban on synthetic colloids in any patient population.

\section{Conclusions}

We cannot support a comprehensive ban on synthetic colloids on the basis of only two trials $[16,17]$ but these two trials represent the best evidence currently available (in terms of randomization, endpoints, and follow-up). Since both suggested that the use of medium-MW HES solutions is associated with harm in patients with septic shock, these 
solutions should not be used routinely for this indication. Synthetic colloids are potent volume expanders but they may be harmful and are probably unnecessary for most patients.

The current data should alert clinicians to potential concerns with the administration of high volumes of HES, particularly the medium-MW HES solutions favoured in many parts of the world. Synthetic colloids definitely should not be used as the sole source of intravenous fluid in critically ill patients, and ordering physicians should be aware of the potential adverse effects, including allergic reactions, renal dysfunction, and coagulopathy. Maximum dosages for these products should be made known to all users and strictly monitored whenever possible. Newer-generation low-MW HES solutions or balanced crystalloid solvents may be safer but this has never been conclusively demonstrated.

\section{Competing interests}

The authors declare that they have no competing interests.

\section{References}

1. McClelland DBL: Safety of human albumin as a constituent of biologic therapeutic products. Transfusion 1998, 38:690-699.

2. Boldt J, Suttner S: Plasma substitutes. Minerva Anestesiol 2005, 71:741-758.

3. Menger MD, Sack FU, Hammersen F, Messmer K: Tissue oxygenation after prolonged ischemia in skeletal muscle: therapeutic effect of prophylactic isovolemic hemodilution. Adv Exp Med Biol 1989, 248:387-395.

4. Barron ME, Wilkes MM, Navickis RJ: A systematic review of the comparative safety of the colloids. Arch Surg 2004, 139:552563.

5. Boldt J, Heesen M, Müller M, Pabsdorf M, Hempelmann G: The effects of albumin versus hydroxyethyl starch solution on cardiorespiratory and circulatory variables in critically ill patients. Anesth Analg 1996, 83:254-261.

6. Boldt J, Schollhorn T, Munchbach J, Pabsdorf M: A total balanced volume replacement strategy using a new balanced hydroxyethyl starch preparation (6\% HES 130/0.42) in patients undergoing major abdominal surgery. Eur $J$ Anaesth 2007, 24:267-273.

7. Boldt J, Müller M, Heesen M, Heyn O, Hempelmann G: Influence of different volume therapies on platelet function in the critically ill. Intensive Care Med 1996, 22:1075-1081.

8. Nascimento P Jr., de Paiva Filho O, de Carvalho LR, Braz JR: Early hemodynamic and renal effects of hemorrhagic shock resuscitation with lactated Ringer's solution, hydroxyethyl starch, and hypertonic saline with or without $6 \%$ dextran-70. J Surg Res 2006, 136:98-105.

9. Winkelmayer WC, Glynn RJ, Levin R, Avorn J: Hydroxyethyl starch and change in renal function in patients undergoing coronary artery bypass graft surgery. Kidney Int 2003, 64: 1046-1049.

10. Kumle B, Boldt J, Piper S, Schmidt C, Suttner S, Salopek S: The influence of different intravascular volume replacement regimens on renal function in the elderly. Anesth Analg 1999, 89: 1124-1130.

11. Palumbo D, Servillo G, D'Amato L, Volpe ML, Capogrosso G, De Robertis E, Piazza O, Tufano R: The effects of hydroxyethyl starch solution in critically ill patients. Minerva Anestesiol 2006, 72:655-664.

12. Dehne MG, Muhling J, Sablotzki A, Dehne KL, Sucke N, Hempelmann G: Hydroxyethyl starch (HES) does not directly affect renal function in patients with no prior renal impairment. $J$ Clin Anes 2001, 13:103-111.

13. Boldt J, Muller M, Mentges D, Papsdorf M, Hempelmann G: Volume therapy in the critically ill: is there a difference? Intensive Care Med 1998, 24:28-36.

14. Sung KC, Suk PK, Ja YM, Ok KK: Effects of intravascular volume therapy using hydroxyethyl starch $(130 / 0.4)$ on postoperative bleeding and transfusion requirements in children undergoing cardiac surgery: a randomized clinical trial. Acta Anaesthesiol Scand 2006, 50:108-111.

15. Upadhyay M, Singhi S, Murlidharan J, Kaur N, Majumdar S: Randomized evaluation of fluid resuscitation with crystalloid (saline) and colloid (polymer from degraded gelatin in saline) in pediatric septic shock. Indian Pediatr 2005, 42:223-231.

16. Schortgen F, Lacherade JC, Bruneel F, Cattaneo I, Hernery F, Lemaire $F$, Brochard L: Effects of hydroxyethylstarch and gelatin on renal function in severe sepsis: a multicentre randomised study. Lancet 2001, 357:911-916.

17. Brunkhorst FM, Engel C, Bloos F, Meier-Hellmann A, Ragaller M, Weiler N, Moerer O, Gruendling M, Oppert M, Grond S, Olthoff D, Jaschinski U, John S, Rossaint R, Welte T, Schaefer M, Kern P, Kuhnt E, Kiehntopf M, Hartog C, Natanson C, Loeffler M, Reinhart K; German Competence Network Sepsis (SepNet): Intensive insulin therapy and pentastarch resuscitation in severe sepsis. N Engl J Med 2008, 358:125-139.

18. Wiedermann CJ: Systematic review of randomized clinical trials on the use of hydroxyethyl starch for fluid management in sepsis. BMC Emerg Med 2008, 8:1-8.

19. Boldt J, Brenner T, Lehmann A, Lang J, Kumle B, Werling C: Influence of two different volume replacement regimens on renal function in elderly patients undergoing cardiac surgery: comparison of a new starch preparation with gelatin. Intensive Care Med 2003, 29:763-769.

20. Cittanova ML, Leblanc I, Legendre C, Mouquet C, Riou B, Coriat $\mathrm{P}$ : Effect of hydroxyethylstarch in brain-dead kidney donors on renal function in kidney-transplant recipients. Lancet 1996, 348:1620-1622.

21. Castro VJ, Astiz ME, Rackow EC: Effect of crystalloid and colloid solutions on blood rheology in sepsis. Shock 1997, 8: 104-107.

22. Schildt $B$, Bouveng $R$, Sollenberg M: Plasma substitute induced impairment of the reticuloendothelial system function. Acta Chir Scand 1975, 141:7-13.

23. Lukasewitz $P$, Kroh $U$, Löwenstein $O$, Krämer $M$, Lennartz $H$ : Quantitative measurement regarding the tissue storage of middle molecular hydroxyethyl starch $200 / 0.5$ in patients with multiple organ failure [in German]. J Anaesth Intensivbeh 1998, 3:42-46.

24. Treib J, Baron JF, Grauer MT, Strauss RG: An international view of hydroxyethyl starches. Int Care Med 1999, 25:258-268.

25. Boldt J: The balanced concept of fluid resuscitation. Brit $J$ Anaesth 2007, 99:312-315.

26. Davidson IJ: Renal impact of fluid management with colloids: a comparative review. Eur J Anaesth 2006, 23:721-738.

27. Schneider M, Valentine S, Clarke GM, Newman MA, Peacock J: Acute renal failure in cardiac surgical patients, potentiated by gentamicin and calcium. Anaesth Intensive Care 1996, 24:647650.

28. Boldt J, Brosch C, Rohm K, Papsdorf M, Mengistu A: Comparison of the effects of gelatin and a modern hydroxyethyl starch solution on renal function and inflammatory response in elderly cardiac surgery patients. Brit J Anaesth 2008, 100:457464.

29. Baron JF: Adverse effects of colloids on renal function. In Yearbook of Intensive Care and Emergency Medicine 2000. Edited by Vincent JL. Berlin: Springer; 2000:486-493.

30. Moran M, Kapsner C: Acute renal failure associated with elevated plasma oncotic pressure. N Engl J Med 1987, 317:150153.

31. Jonville-Bera AP, Autret-Leca E, Gruel Y: Acquired type I von Willebrand's disease associated with highly-substituted hydroxyethyl starch. N Engl J Med 2001, 345:622.

32. De Jonge $E$, Levi M: Effect of different plasma substitutes on blood coagulation: a comparative review. Crit Care Med 2001, 29:1261-1267.

33. Zander R, Boldt J, Engelmann L, Mertzlufft F, Sirtl C, Stuttmann R: The design of the VISEP trial: critical appraisal [in German]. Anaesthesist 2007, 56:71-77.

34. Sakr Y, Payen D, Reinhart K, Sipmann FS, Zavala E, Bewley J, Marx G, Vincent JL: Effects of hydroxyethyl starch administration on renal function. Br J Anaesth 2007, 98:216-224.

35. Bernal W, Wendon JA: Hydroxyethylstarch as a risk factor for acute renal failure in severe sepsis. Lancet 2001, 358:583. 
36. Mahmood A, Gosling P, Vohra RK: Randomized clinical trial comparing the effects on renal function of hydroxyethyl starch or gelatine during aortic aneurysm surgery. Br J Surg 2007, 94:427-433.

37. Godet G, Lehot JJ, Janvier G, Steib A, De Castro V, Coriat P: Safety of HES 130/0.4 (Voluven(R)) in patients with preoperative renal dysfunction undergoing abdominal aortic surgery: a prospective, randomized, controlled, parallel-group multicentre trial. Eur J Anaesthesio/ 2008, 20:1-9.

38. Kozek-Langenecker SA, Jungheinrich $C$, Sauermann W, Van der Linden P: The effects of hydroxyethyl starch 130/0.4 (6\%) on blood loss and use of blood products in major surgery: a pooled analysis of randomized clinical trials. Anesth Analg 2008, 107:382-390.

39. Haisch G, Boldt J, Krebs C, Kumle B, Suttner S, Schulz A: The influence of intravascular volume therapy with a new hydroxyethyl starch preparation (6\% HES 130/0.4) on coagulation in patients undergoing major abdominal surgery. Anesth Analg 2001, 92:565-567

40. Blasco V, Leone M, Antonini F, Geissler A, Albanèse J, Martin C: Comparison of the novel hydroxyethylstarch 130/0.4 and hydroxyethylstarch 200/0.6 in brain-dead donor resuscitation on renal function after transplantation. $\mathrm{Br} J$ Anaesth 2008, 100: 504-508. 